\section{DISINFECTANT SPRAY}

Kemdent has introduced Practicesafe disinfectant spray as part of their new range of cross infection products. If you spend $\mathfrak{£} 20$ on any Kemdent cross infection control product by the end of June you will receive a 20\% discount.

Practicesafe can

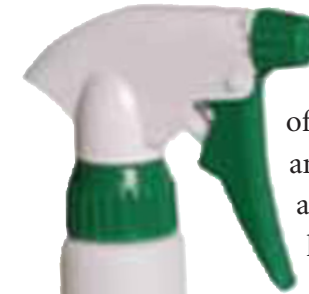

disinfect the alcohol resistant surfaces of a dental practice and clean hand and angle pieces to a very high standard. It is highly effective and without an overpowering fragrance.

Telephone 01793770256 or visit www.kemdent.co.uk.

\section{RAPID RENOVATION}

Renovating flooring in a dental practice can be a noisy, dusty job which requires treatment rooms to be put out of action while the work is completed. Now a special adhesive - Sigan 3 - allows floors to be renovated without creating dirt or noise, or interrupting treatment. It enables various elastic coverings, such as PVC and carpeting, to be bonded directly to existing artificial stone floor. Fitted carpet can be laid on existing flooring or laminate. The adhesive does not have any toxic effects and there are no unpleasant odours during, or after, the laying of the new floor. The adhesive can also withstand high loads, and can be removed without leaving any residues behind.

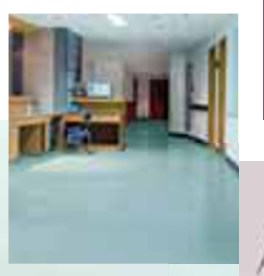

1

\section{INSTRUMENTS FOR ALL}

Dentsply is aware that hygienists need a range of instruments for delicate and complex work. It produces a variety of instruments with three unique handle designs.

Flexichange comprises a wide range of periodontal instruments with hourglass shaped silicone handles. The mid-range handle launched last year, Artio, offers a range of 60 periodontal and restorative instruments on lightweight resin handles with strong steel, sharp cutting edges.

For the budget conscious consumer the Lustra range offers a change of instrument shapes with metal handles.

For more information telephone 08000723313 or email enquiry@ dentsply-gb.com.

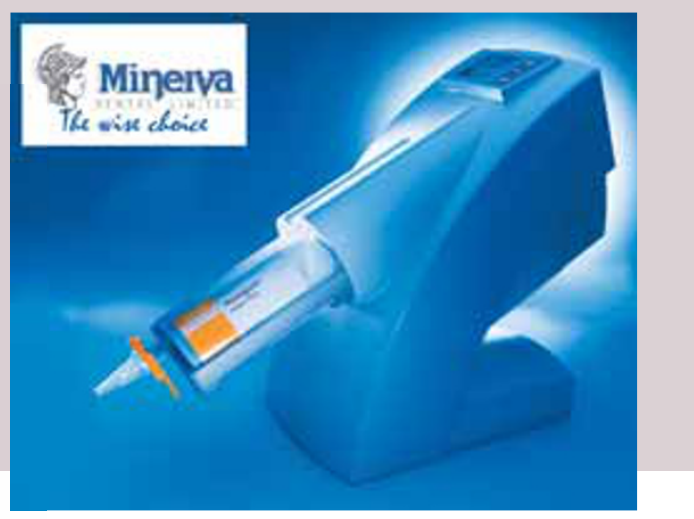

\section{BESPOKE FURNITURE}

Are you planning to renovate your reception or surgery areas? Classic Dental Cabinetry offers a range of bespoke cabinetry, stools and reception furniture. It is available on a supply only basis, or with full installation support.

The cabinetry range features quality worktops, PVC-edged doors and carcass fronts, quality sinks and mixer taps - all designed on a bespoke basis. Split height designs and custom built instrument drawers can also be supplied.

The reception furniture is supplied in a split level design and complies with disabled access requirements. Tailor made filing systems are also available.

Telephone 01207570321 or info@classic dentalcabinetry.co.uk or visit www.classic dentalcabinetry.co.uk.

\title{
$\lambda$
}
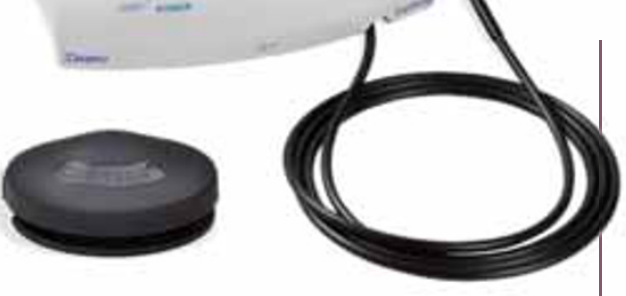

\section{COMFORTABLE CLEAN}

Make sure patients return to the practice by ensuring procedures are as comfortable as possible. When it comes to probing, scaling and root planing (SRP) procedures, comfort is very important as repeat visits are involved.

Dentsply has found a winning combination: Cavitron Plus, Cavitron Slimline insert tips and Oraquix, the needle free anaesthesia indicated for SRP procedures. If you would like a demonstration of the Cavitron Plus in your practice, or a closer look at Oraquix, telephone 01932853422.

\section{PERFECT MIXES}

Minerva Dental has introduced a new fully automatic mixing machine, MixStar-eMotion. Its three, electronically controlled speed settings ensure consistent results even with more viscous materials.

Setting and working time in the mouth can be adjusted with two timers and saved on the programmes. The mixing process can be started by pressing a button, or by using a foot operated switch, which can be plugged in for hands free operation. With the aid of a serial interface, software can be updated any time via the internet.

Extremely user-friendly, the MixStar-eMotion is designed to deliver perfectly mixed, homogeneous, bubble free impression material directly into the impression tray or syringe. Telephone 02920442800. 